\title{
Inability of Monoclonal Anti-light Chain Antibody to Detect Clonal B-Cells in a Patient with Follicular Lymphoma by Multicolor Flow Cytometry
}

\author{
Jeroen F. van Velzen, ${ }^{*}$ Dorine van den Blink, Ingrid E.H. Wiegers, \\ and Andries C. Bloem \\ Section of Medical Immunology, Laboratory of Translational Immunology, \\ University Medical Centre Utrecht, Utrecht, The Netherlands
}

\begin{abstract}
Background: Our recent publication "Inability of a monoclonal anti-light chain antibody to detect clonal plasma cells in a patient with multiple myeloma by multicolor flow cytometry," underlined the importance of choice of antibodies to detect cytoplasmic light chains. Our present study extends this finding for detection of surface immunoglobulin (Slg) light chains on clonal B-cells. Methods: Multicolor flow cytometry was used for analyzing bone marrow (BM) from a patient with a CD10-positive follicular lymphoma for infiltrating clonal B-cells. Results: In the BM aspirate, B cells could be identified ex-
\end{abstract}

pressing CD19, CD10, and high levels of CD20. No Slg light chain expression was found on this population of B cells employing monoclonal antibodies. Re-analysis using polyclonal antibodies against Slg light chains, revealed presence of lambda light chains on the CD10positive B-cells. Conclusions: These data illustrate when antibodies against SIg light chains are employed for B-cell clonality assessment, polyclonal antibodies are preferred over monoclonal antibodies. J. Clin. Lab. Anal. 28:493-495, 2014. (c) 2014 Wiley Periodicals, Inc.

\section{INTRODUCTION}

In our recent publication "Inability of a monoclonal anti-light chain antibody to detect clonal plasma cells in a patient with multiple myeloma by multicolor flow cytometry," we showed that the choice of antibodies is important for the outcome of flow cytometric cytoplasmic clonality assessment of plasma cells (1). We concluded that for clonality assessment of plasma cells the use of polyclonal antibodies over monoclonal antibodies is preferred for the detection of intracellular immunoglobulin light chains.

In this case report, we extend this finding to the detection of surface-bound monoclonal immunoglobulin in case of a patient with a B-cell non-Hodkin lymphoma with bone marrow (BM) localization. Non-Hodgkin lymphoma is the most common blood cancer; therefore this information is relevant for all laboratory specialists that employ flow cytometry for the routine analysis of blood and $\mathrm{BM}$ specimens for the presence of mature monoclonal $\mathrm{B}$ cells.

\section{METHODS}

The flowcytometric analysis was performed on heparinized BM from a patient with an established CD10positive follicular lymphoma. For the analysis, whole BM cells $\left(1 \times 10^{6}\right)$ were extensively washed to remove circulating immunoglobulins and rabbit serum (Beckman Coulter, Miami, FL) was used as a blocking agent during incubation with titrated monoclonal antibodies directed against CD19 PercP-Cy5.5 (clone SJ25C1), CD10 PE-Cy7 (clone HI10a), kappa/lambda simultest (kappa-FITC/lambda PE clones TB28-2, and 1-155-2,

\footnotetext{
* Correspondence to: Jeroen F. van Velzen, Section of Medical Immunology, Laboratory of Translational Immunology, University Medical Centre Utrecht, Heidelberglaan 100, 3584 CX Utrecht, The Netherlands. E-mail: j.vanvelzen@umcutrecht.nl
}

Received 16 July 2013; Accepted 28 October 2013

DOI $10.1002 /$ jcla.21716

Published online in Wiley Online Library (wileyonlinelibrary.com). 
A

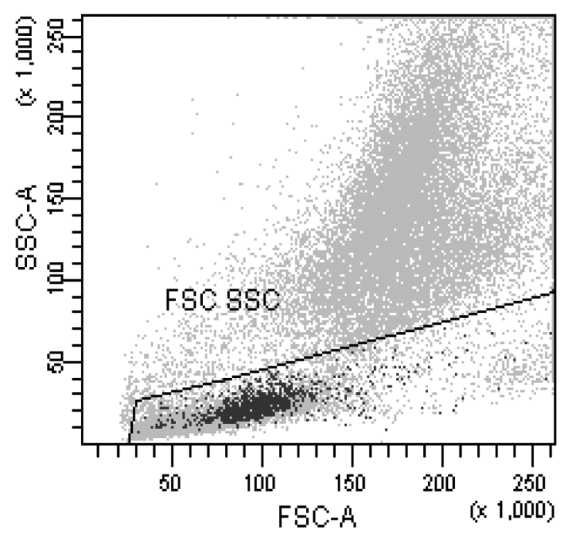

B

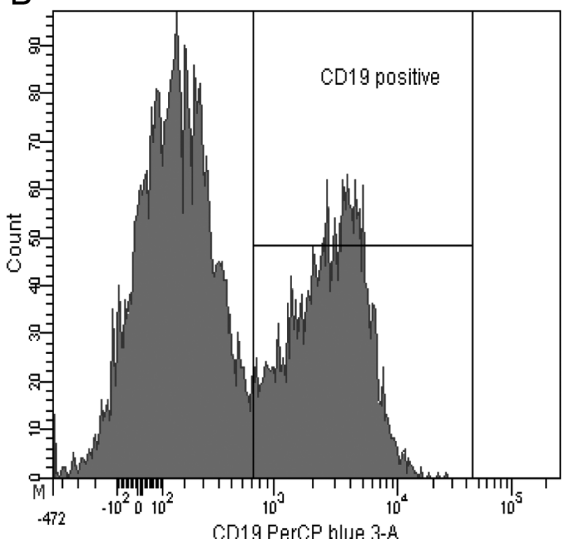

C

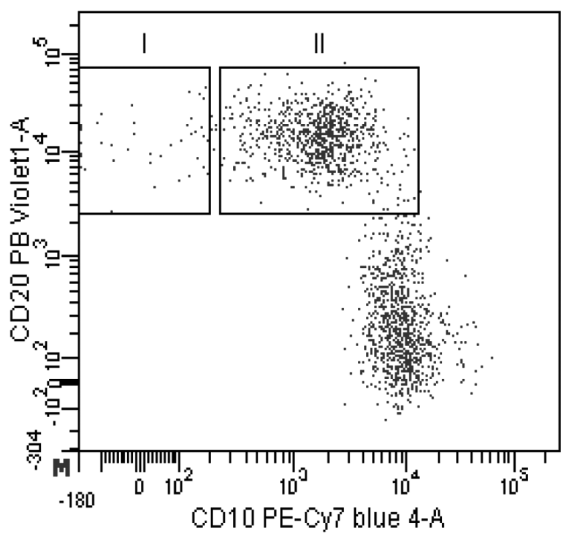

D

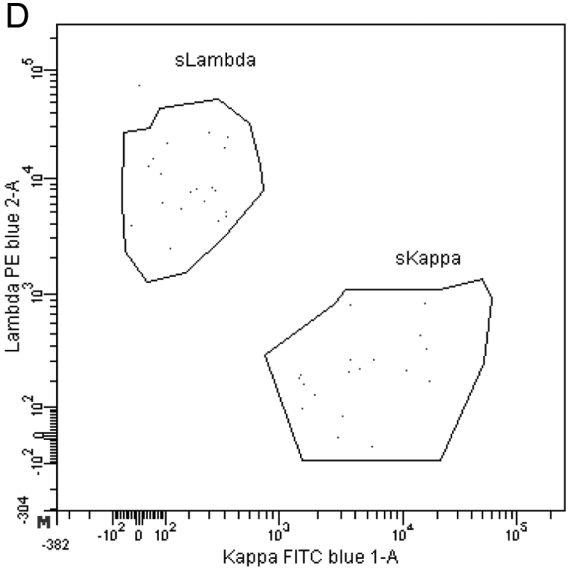

F

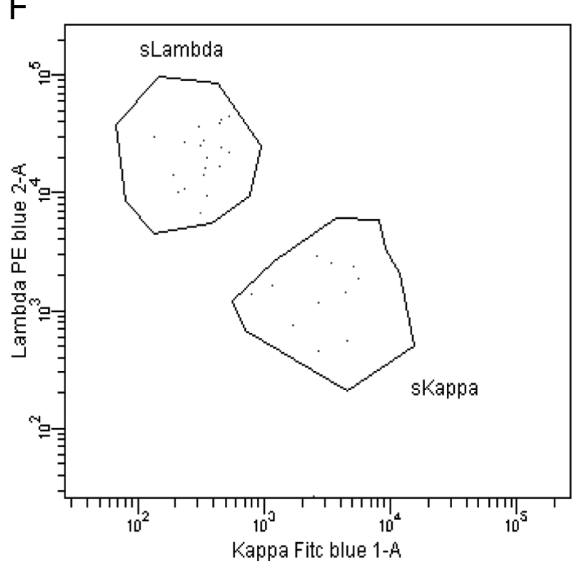

E

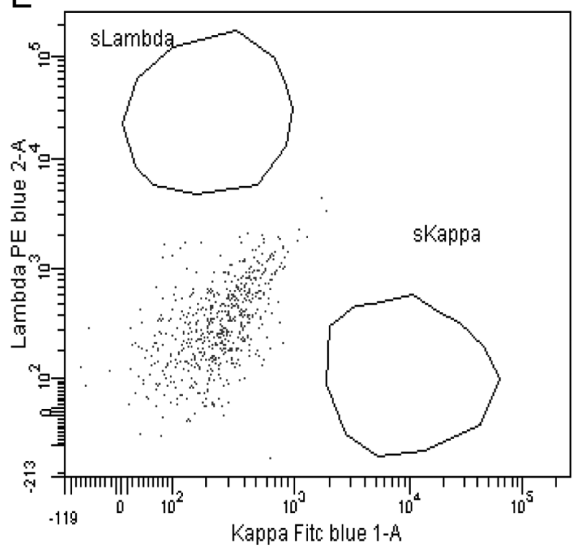

G

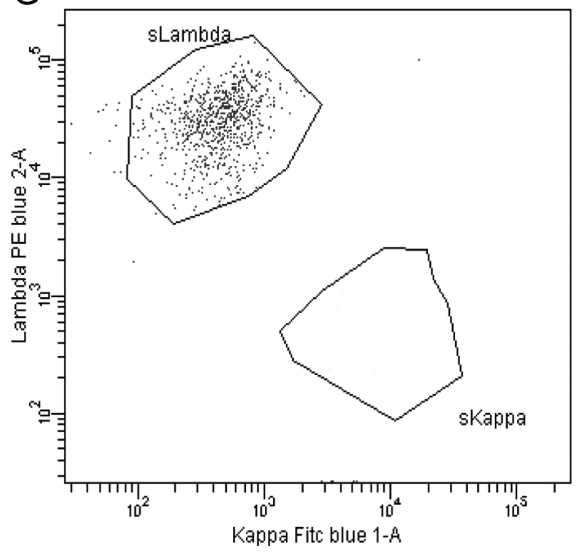

Fig. 1. Characterization of B cells. B cells are identified based on sequential gating on FSC and SSC characteristics (A) and CD19 positivity (B). Three B-cell subpopulations are discerned based on CD10 and CD20 expression (C). Subpopulation I is positive for CD20 and negative for CD10. Subpopulation II is positive for CD10 and CD20 and a population of progenitor B cells is shown, which do not express surface light chains (data not shown). Population I is positive for surface kappa or lambda light chains using monoclonal antibodies (D) or polyclonal antibodies (F). Subpopulation II does not stain for surface kappa or lambda chains using monoclonal antibodies (E), but is positive for surface lambda light chain when stained with polyclonal antibodies.

respectively), all from Becton Dickinson and CD20 eFluor450 (clone 2H7) from eBioscience (San Diego, CA). Incubation was performed for $15 \mathrm{~min}$ at room temperature in the dark. Subsequently, the cells were washed and immediately analyzed on a 3-laser Canto II flow cytometer (Becton Dickinson, Mountain View, CA). Compensation and instrument set-up were performed as described before (1). 


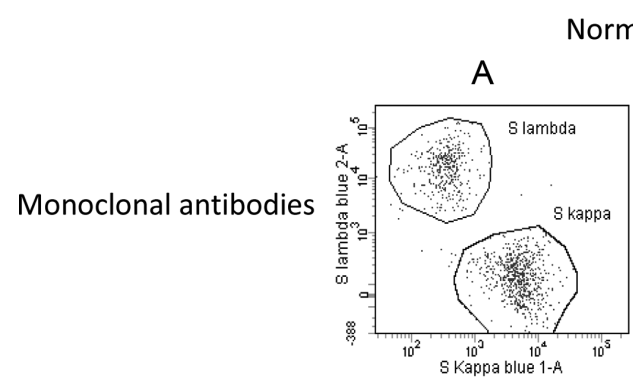

Normal donor
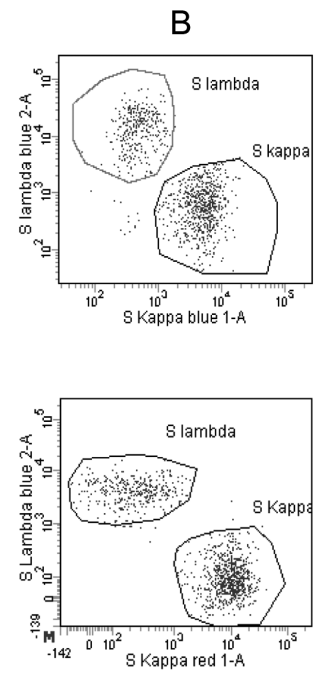
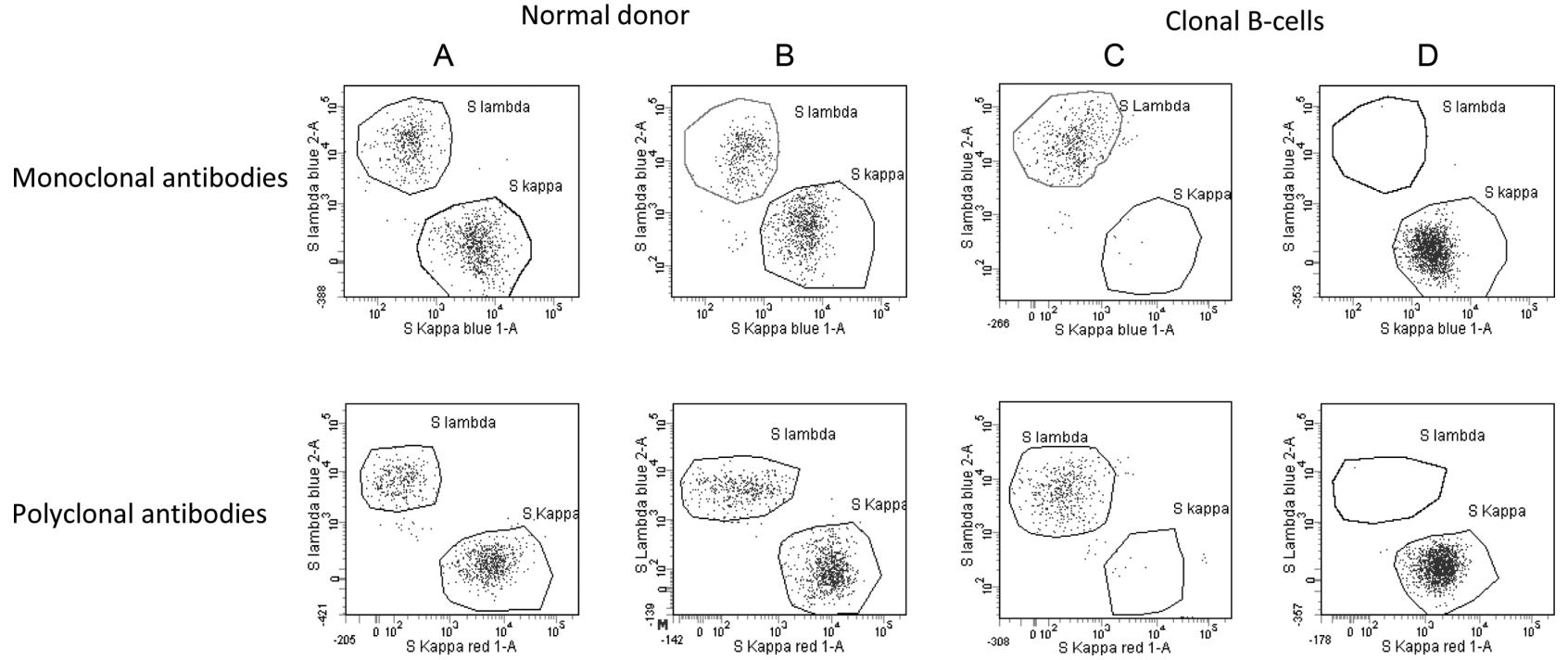

Fig. 2. Control and patient samples. Mono- and polyclonal antibodies were used to stain surface kappa and lambda light chains on CD19 positive B cells present in blood samples of two healthy donors (A and B) and two patients with a B-cell non-Hodgkin lymphoma (C and D).

\section{RESULTS AND DISCUSSION}

In Fig. 1, results of the flow cytometric analysis are shown. B cells are selected based on sequential gating on FSC and SSC characteristics and CD19 expression (Fig. 1A and B). Based on the differential expression of CD10 and CD20 within the B-cell compartment (Fig. 1C), a population I $\left(\mathrm{CD} 10^{-} \mathrm{CD} 20^{+}\right)$and $\mathrm{II}\left(\mathrm{CD} 10^{+} \mathrm{CD} 20^{+}\right)$ can be discerned. The third population, which expresses CD10 but lacks CD20, is of immature progenitor B-cells (data not shown). Analysis of surface immunoglobulin (SIg) light chains using monoclonal antibodies revealed polyclonality in B-cell population I and absence of surface kappa or lambda expression on B-cell population II. The presence of surface light chains on B-cell population I excludes a staining artifact.

In normal $\mathrm{BM} \mathrm{CD} 10^{+}$progenitor, $\mathrm{B}$ cells include pro$\mathrm{B}$, pre-B, and immature transitional B-cells (2); immature transitional B-cells express CD20 and SIg levels comparable to mature B cells. Since population I and II have comparable levels of CD20, we questioned the absence of SIg light chains on population II and repeated the staining with polyclonal rabbit $\operatorname{IgG} \mathrm{F}\left(\mathrm{ab}^{\prime}\right)_{2}$ kappa FITC and polyclonal rabbit $\operatorname{IgG~} \mathrm{F}\left(\mathrm{ab}^{\prime}\right)_{2}$ lambda PE (FR0481 multimix, DakoCytomation, Heverlee, Belgium). The results in Fig. 1F and G show an expression of membranebound kappa and lambda light chains on B-cell population I and membrane-bound lambda light chains on B-cell population II, indicative for an infiltration of clonal
B-cells positive for membrane-bound lambda light chains, CD20 and CD10. This finding suggests that the epitope recognized by the monoclonal antilambda light chain antibody is not expressed or accessible on the SIg light chain of the aberrant B-cell population. In Fig. 2, SIg light chain analyses on additional peripheral blood samples from two normal individuals (A and B) and two patients with a B-cell non-Hodgkin lymphoma (C and D) are shown. In these samples, no differences were observed in staining outcome between the monoclonal and polyclonal antibodies.

In conclusion, these data illustrate that in some patient samples the use of monoclonal antibodies for clonality assessment based on light chain distribution analysis on membrane-bound immunoglobulins of B cells can lead to false-negative results, which might have clinical impact. Therefore, the use of polyclonal antibodies over monoclonal antibodies is preferred for the detection of membrane-bound immunoglobulin light chains.

\section{REFERENCES}

1. van Velzen JF, van den BD, Bloem AC. Inability of a monoclonal anti-light chain antibody to detect clonal plasma cells in a patient with multiple myeloma by multicolor flow cytometry. Cytometry B Clin Cytom 2013;84:30-32.

2. van GR, van Tilburg CM, Nibbelke EE, et al. Refined characterization and reference values of the pediatric T- and B-cell compartments. Clin Immunol 2009;133:95-107. 